\title{
DIRECTIONAL ANTENNA BASED EFFICIENT LOCATION AWARE ROUTING IN MOBILE ADHOC NETWORK
}

\author{
G. Jayasree, K.P. Indulekha and B. Malarkodi \\ Department of Electronics and Communication Engineering, National Institute of Technology, Tiruchirappalli, India
}

\begin{abstract}
Mobile Adhoc Network (MANET) also called as wireless ad hoc network is a self-organizing, self-configuring infrastructure less network containing a group of mobile nodes communicating wirelessly. As the hosts move often resulting in dynamic topology of the network, routing seeks more attention. Therefore, routing protocol using node's location information like LAR (location aided routing) has emerged as potential solution. Here, the route discovery is limited to a small region named as request zone in contrast to blind flooding over the entire network. Also it is noticeable that the shape and size of the request zone play a vital role in enhancing the protocol's performance. After various analyses it was concluded that for higher node density, elliptical shaped request zone performs better than other possible shapes. Further, suitable route must be chosen based on current load status of the network so that successful delivery of packets is ensured. Generally, omni-directional antennas are used for communication between moving motes. The disadvantage of mobile ad hoc networks with omnidirectional antenna lies in the limited capacity caused by high interference and low spatial reuse. This paper focuses on obtaining optimal size for request zone in accordance with varying node density. Further, optimal path between source and destination is selected using Dijkstra's algorithm. Our simulation results show that directional antennas outshines the performance of omni-directional antennas in increasing transmission range of nodes, reducing the number of redundant nodes involving in data communication etc.
\end{abstract}

Keywords:

MANETs, Request Zone, Directional Antennas, Routing Overhead, Location Aided Routing

\section{INTRODUCTION}

A MANET is an autonomous system of moving motes, where each mote acts both as a router and an end host. It is specifically named as Ad hoc as it does not depend on centralized framework such as routers in wired networks or access points in infrastructured wireless systems. These systems are self-arranging, dynamic systems in which motes are allowed to move. These networks do not have the complexities of framework setup and organization, empowering devices to make and join systems "on the fly" anyplace, anytime [37]. These networks are majorly deployed by community users such as military, researchers, business, educational institutes and emergency services. Every node in a MANET is allowed to move autonomously towards any direction, and henceforth alters its links to other nodes frequently. Individual nodes hold responsibility for discovering other nodes, with which they can communicate directly. Because of the limited signal transmission range of each node, not all motes can directly communicate with each other. Along these lines MANET faces many challenges. One of such biggest challenge is routing. Routing is a process of choosing a path in the network for transmission of information. An Ad hoc routing protocol is a standard that controls how a node selects a path to route data between moving devices [7] [38]. One such routing protocol which utilises location information in determining the route is called Location Aided Routing (LAR) [2] [14]. In network implementing LAR, nodes obtain geographical location of other nodes by localization schemes such as Global Positioning System (GPS). One of the major problem with the existing LAR is, shape of its request zone is not producing optimized results in varying node densities. If we define smaller request zones, it leads to reduction in overhead but affects successful packet delivery and delay. Also, there is a possibility of holes inside the request (forwarding) region. If we define larger request zones it enhances the probability for successful route finding but it can increase the routing overhead. Extending the size of the request zone to the entire network after the failure of route discovery in first attempt will lead to high control overhead, wastage of bandwidth. Hence, in this paper we discussed about resizing of request zone in multistep instead of blind flooding [3]. Further, usage of omnidirectional antennas for transferring data leads to redundant packet transmissions [4].

Henceforth, this paper proposes the Modified LAR with optimal shapes of request zones according to varying node densities. Furthermore, we use directional antennas to enhance the protocol's performance after the optimal path between source and destination is chosen.

This paper is organized as follows: section 2 discusses related background material, motivated factors of this work and contributions. In section 3 various shapes and resizing of request zones of LAR are presented. Section 3 also discusses about route finding algorithms and directional antennas. Simulated results showing comparison of omni-directional and directional antennas, network parameters corresponding to varying densities of nodes in different shapes, sizes of Request zones are analyzed in section 4. Finally section 5 presents conclusions.

\section{REVIEW OF ROUTING PROTOCOLS IN MANET}

In the course of recent years, many routing protocols have been proposed in MANET [7], [13]. They can be grouped into two categories to be specific, topology-based routing and positionbased routing protocols [6]. Topology based routing protocols direct packets based on network links information whereas position-based routing protocols utilizes physical data about the engaged motes. Topology based routing can be further arranged into proactive, reactive and hybrid routing protocols. In proactive protocols, the network links are resolved long before routing and in reactive protocols it is done at the time of routing. Hybrid protocols incorporate both proactive and reactive routing. In the position based routing protocols, location information about the receiver node is known and utilized. Further, they are divided into 
greedy forwarding and restricted flooding. Motes transfer data to those nodes which are best in progress in greedy forwarding. Preferably, this procedure is repeated until the destination mote is reached. However route discovery is not present in greedy forwarding. In contrast, messages are broadcasted to a chose number of motes which are located closer to the destination in restricted flooding. Thus probability of packet collisions which results due to rebroadcast of messages by nodes using same transmission channel, is reduced and energy consumption is also lowered.

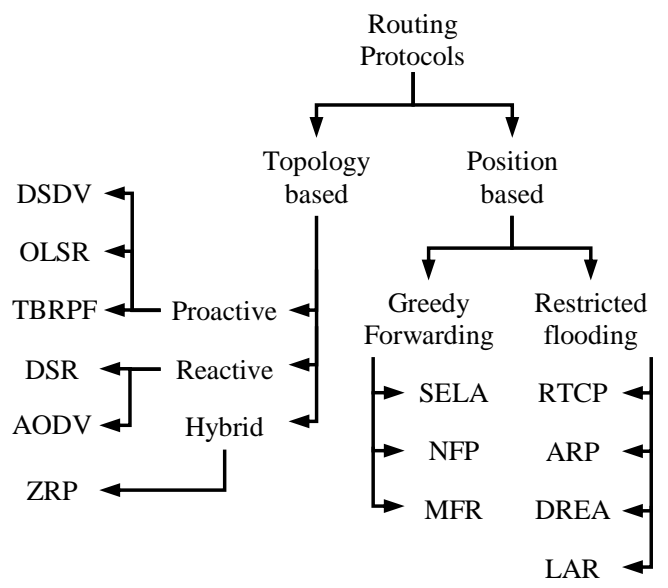

Fig.1. Classification of routing protocols in MANET

\subsection{MOTIVATION AND CONTRIBUTIONS}

Location Aided Routing (LAR) protocol divides the entire network into two geographical zones namely request zone and expected zone. The existing LAR uses a rectangular request zone containing the expected zone i.e. circular region around destination node and source node location [1]. In route discovery phase, Route request (RREQ) packets are flooded within the defined rectangular request zone. RREQ packets are propagated further, by the nodes residing inside the request zone and discarded by the other nodes. However, if path to destination is not found through this restricted flooding, RREQ packets are flooded over the entire network. Hence request zone shape and size must be chosen with caution. Defining narrow shaped request zones will lessen the control overhead but it decreases the rate of successful packet delivery and increases latency. Likewise, there is a chance of existence of holes within the restricted request zones. In contrary bigger request zones improves the probability of successful path discovery but they increment control overhead. Furthermore, omni-directional antenna at the sender node transmits data in all directions resulting in the unnecessary receptions at neighboring nodes [4], [12]. This increases packet collisions, interference and delay in the network. Conversely directional antennas are capable of directing electromagnetic waves in specific direction. Thus they reduce the packet loss as well as improve the coverage range. Motivated by the above facts, this paper presents us the optimal shape and size of the request zone in accordance with varying densities of nodes in the network. Plot of routing overhead occurred while employing various shapes of request zones will further support the obtained conclusions. Additionally, network scenarios with the employment of omni-directional antennas and directional antennas has been compared and analysed. Also determination of optimal route considering the current load on network links has been presented.

\section{MODIFIED LAR WITH DIRECTIONAL ANTENNAS}

LAR divides the entire network into two geographical regions namely Request zone and Expected zone [1]. Request zone is defined as the area within which the route request packets are allowed to be forwarded. Expected zone is the region in which the destination node is expected to be present.

\subsection{RECTANGULAR REQUEST ZONE}

The request zone is defined as a small rectangle which includes the current location $\left(X_{p}, Y_{p}\right)$ of-course $\mathrm{P}$ and the expected zone. Let us assume that source knows the destination $Q$ location $\left(X_{q}, Y_{q}\right)$ at time $T_{0}$ and average speed of $Q$ is $U$. Now at time $T_{1}, P$ can form the expected zone as a circular region having center $(C)$ and radius $(R)$ as follows:

$$
\begin{aligned}
\text { Center }(C) & =\left(X_{q}, Y_{q}\right) \\
\text { Radius }(R) & =U\left(T_{1}-T_{0}\right)
\end{aligned}
$$

Then $P$ broadcasts the RREQ packets which contains the information about four corner coordinates of the request zone and $\left(X_{q}, Y_{q}\right)$. Node which receives this packet will rebroadcast it if it resides inside the rectangular request zone otherwise ignores it. This is rehearsed until the point where $Q$ receives the RREQ packet. Then it sends a Route Reply (RREP) packet that incorporates its present location and current time. Upon receiving RREP packet, mote $P$ will note the location of $Q$ and the related time for future purposes.

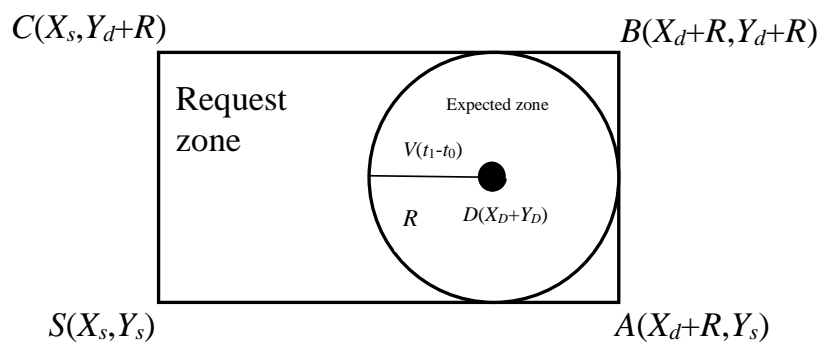

(a) Source node $S$ outside the Expected Zone

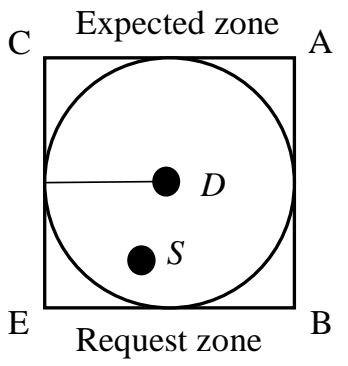

(b) Source node $S$ inside the Expected Zone

Fig.2. Rectangular request zone

\subsubsection{Size of the Request Zone:}

Note that the size of the rectangular request zone above is proportional to

- Average speed of movement $U$ 
- Time elapsed since the last known location of the destination was recorded

In our implementation, the sender comes to know location of the destination only at the end of a route discovery at low speeds, route discoveries occur after long intervals, because routes break less often (thus $T_{1}-T_{0}$ is large). So, although factor (a) above is small factor (b) becomes large at low speeds, potentially resulting in a larger request zone. At high speeds as well, for similar reasons, a large request zone may be observed. So, in general, a smaller request zone may occur at speeds that are neither too small, nor too large. For low speeds, it is possible to reduce the size of the request zone by piggybacking the location information on other packets, in addition to route replies.

\subsubsection{Multi-Step Increase of the Request Zone:}

To enhance the performance of LAR in terms of routing overhead, we should construct an appropriate request zone. Henceforth, in this paper, we propose adaptive-sizing of the request zone aiming to upgrade the probability of finding optimal route by reducing control overhead, loss of data packets. We propose that the source node re-computes an "enlarged" request zone, in case of failure of the route discovery phase, instead of going for flooding. This process can be repeated $n$ times before switching to flooding; so called as n-step or multi-step [5]. Our idea is to make use of the rectangular request zone defined by LAR scheme [26] at the first attempt of route discovery and in case of failure, if the destination is not found, to increase the request zone instead of flooding route requests in the whole network. If the successive route discovery fails as well, the protocol re-starts with a new increase of the request zone, and so on for every failure of the route discovery. This procedure proceeds to $n$-steps. The increased request zone computed at each step is rectangular as well, as shown in Fig.3. The dimensions of the increased rectangle are function of the destination position known by the source at time $T_{0}$. The increments $\Delta X_{n-s t e p}$ and $\Delta Y_{n-}$ step of the request zone has to satisfy the following constraints:

$$
\begin{aligned}
& \Delta X_{n \text {-step }}>\Delta X_{\text {min }}-n=(n-1) \times T_{\text {timeout }} \times V_{\text {max }} \times \delta x \\
& \Delta Y_{n \text {-step }}>\Delta Y_{\text {min }}-n=(n-1) \times T_{\text {timeout }} \times V_{\text {max }} \times \delta y
\end{aligned}
$$

where $\delta x$ and $\delta y$ are error factors for the evaluation of node $\mathrm{Q}$ position $\left(X_{q}, Y_{q}\right), V_{\max }$ is the maximum velocity of node $Q$, and $T_{\text {timeout }}$ is the timeout for successive route requests (this means that if a route reply packet is not received within the $T_{\text {timeout }}$ period, then a second route request packet is sent in the increased request zone). In this way, the increment of the request zone guarantees the covering of the area in which the destination can be found.

At the $n^{\text {th }}$ step, the request zone is increased as follows:

$$
\begin{aligned}
& \Delta X_{n \text {-step }}=X_{(n-1) \text {-step }}+\Delta X_{n \text {-step }} \\
& \Delta Y_{n \text {-step }}=Y_{(n-1) \text {-step }}+\Delta Y_{n \text {-step }}
\end{aligned}
$$

where,

$$
\begin{aligned}
& \Delta X_{n \text {-step }}=P_{x-n}+X_{(n-1) \text {-step }} \\
& \Delta Y_{n \text {-step }}=P_{y-n}+Y_{(n-1) \text {-step }}
\end{aligned}
$$

$X_{n \text {-step }}, Y_{n \text {-step }}$ are the horizontal and vertical sizes of the request zone respectively at the $n^{\text {th }}$ step; $P_{x n}, P_{y n}$ are percentages of $X_{(n-}$ 1)step, $Y_{(n-1) \text { step }}$ respectively. Further $\Delta X_{n \text {-step }}, \Delta Y_{n \text {-step }}$ satisfy constraints Eq.(2) and Eq.(3) respectively.

Briefing the steps involved in route discovery by the multistep LAR are the following:
- First route discovery attempt inside the rectangular request zone defined by original LAR scheme 1.

- In case of failure, second or successive route discovery attempts inside the increased rectangular request zone.

- In case of $n^{\text {th }}$ failure, $n^{\text {th }}+1$ route discovery attempt through flooding in the whole network.

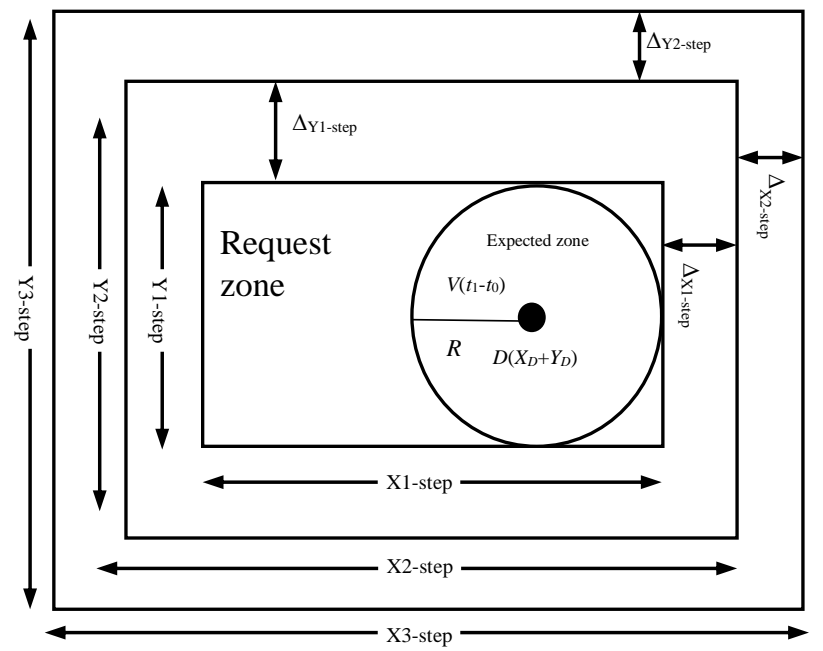

Fig.3. Multistep increase of rectangular zone

\subsection{TEARDROP SHAPED REQUEST ZONE}

As the name suggests, here request zone is in the shape of Teardrop. It includes $\triangle P C D$ and the expected zone [1]. Here, at points $C, D$ the tangent lines from source $(P)$ will touch the expected zone. Expected zone is formed as a circle with radius $U \times\left(T_{1}-T_{0}\right)$ and centre $\left(X_{q}, Y_{q}\right)$.

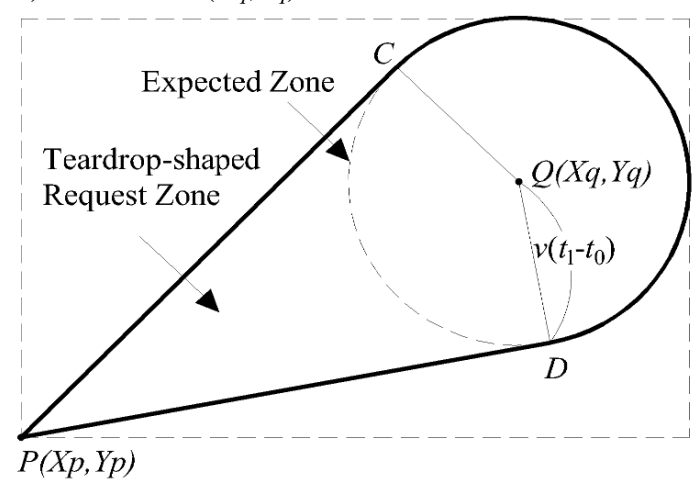

Fig.4. Teardrop shaped request zone

We define the expected zone to be the circular area with radius $U \times\left(T_{1}-T_{0}\right)$ centered at location $\left(X_{q}, Y_{q}\right)$ of the destination node. In the case where $U$ or $T_{1}-T_{0}$ is small, a small expected zone is determined, and then the teardrop-shaped request zone is also small. Too small a request zone results in the failure of the route discovery process. The radius and center of the extended expected zone are $R_{0}$ and $\left(X_{q}, Y_{q}\right)$ respectively. The radius $R_{0}$ is calculated by following equation:

$$
R_{0}=R \times f(n, \theta)
$$

In Eq.(8), $R$ and $\theta$ are the transmission range and the angle of radiation of the directional antenna, respectively, and $n$ is the number of neighbouring nodes. We assume that $\theta$ is not variable 
but fixed. Each node can estimate $n$ by simply overhearing any transmitted packets in its own vicinity. The function $f$ should be determined based on factors such as the network mobility and node density. Here we use the following formula:

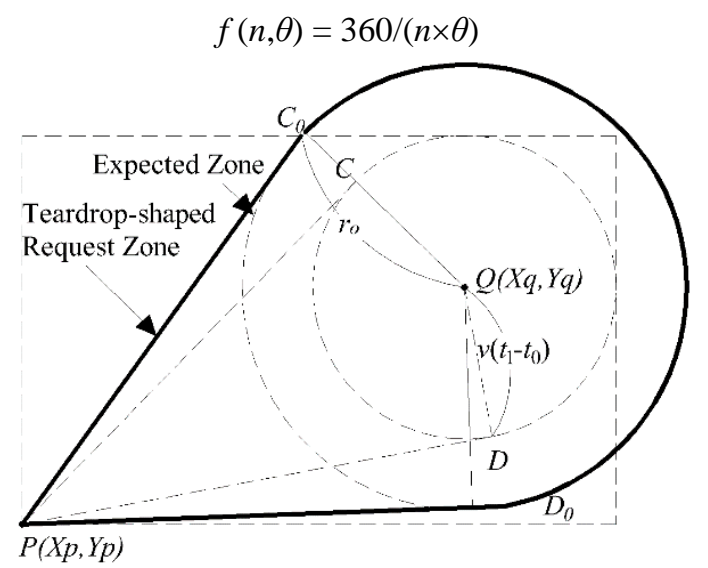

Fig.5. Resizing of tear drop shaped zone

In Fig.5, $C_{0}$ and $D_{0}$ are the contact points of the tangent lines from $P$ to the extended expected zone. If $U \times\left(T_{1}-T_{0}\right)$ is equal to or less than $R_{0}$ then the teardrop shaped area consists of $\triangle P C_{n} D_{n}$ and the extended expected zone, instead of $\triangle P C D$ and the expected zone.

\subsection{TRIANGULAR REQUEST ZONE}

The request zone formed is triangular in shape (smallest isosceles triangle). It includes the present location of $\mathrm{P}$ and the expected zone. Triangular shape of the request zone is obtained by points $P, C, B$ where $P$ is the location of source $\left(X_{p}, Y_{p}\right)$ and $C$, $B$ are tangent points on the expected zone. Advantages of going for a triangular zone are that it enables to forward route request packets in a narrower space [2]. Thus the request is forced to be propagated as straight to destination as possible. Indirectly, it enables a higher chance to select a shorter route. One can observe that the area of triangle zone is less compared to the rectangular zone area defined irregular LAR scheme.

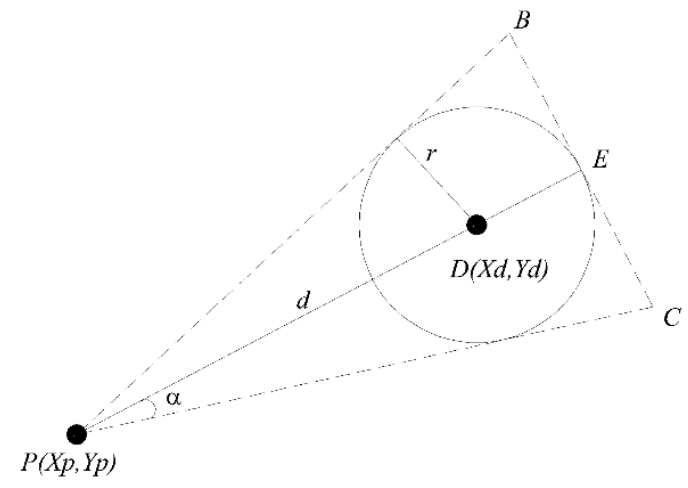

Fig.6. Triangular Request Zone

As shown in the Fig.6 triangular request zone is formed with radius as $U \times\left(T_{1}-T_{0}\right)$ and $x$ is the distance between $P$ and point $E$. Angle $\alpha$ is given by

$$
\alpha=\sin ^{-1}\left(\frac{r}{x}\right)
$$

\subsubsection{Resizing of Request Zone:}

During the failure of route discovery in first round of process, expanding it to the entire network will devalue the benefits of restricted request zone. Therefore, to solve this problem the following approach is proposed. When a transmitter using a Triangle Zone in last routing process is unable to discover the route to a receiver within a timeout interval, it expands the Triangle Zone by increasing the search angle with an angle increment and initiates a new route discovery until the search angle exceeds an angle threshold. The Fig.7 shows Triangle Zone PEG is expanded by increasing search angle in the rerouting process. The Fig.5 shows triangle zone PEG is expanded to PEG $G^{\prime}$ by increasing search angle from $\alpha$ to $\alpha^{\prime}$ in the rerouting process. If a route is not discovered when the search angle exceeds a threshold, the sender mote expands the request zone to the entire network and reroutes again [2]. Let's use the example in Fig.7 to show how it works. In the first attempt, $P$ uses a triangle PEG as a request zone and initiates a route request with sequence number 1. There are three forwarding nodes, $I_{1}, I_{2}$ and $I_{3}$ in this triangles 'one. Assume that $I_{3}$ is out of the transmission range of node $P, I_{1}$ and $I_{2}$. Hence, only $I_{1}, I_{2}$ can forward the request from $P$ and record this event in their seen table. There is no other node can assist for relaying the request to the destination, so that the destination will not emit a route reply packet. Because $S$ does not receive a route reply from destination $D$ in the specified time interval, it claims that the first route discovery fails and retries a route discovery. If the last search angle a does not exceed the threshold, $P$ enlarges the search angle to $\alpha^{\prime}$ and resends a route request with the same sequence number (i.e. 1). In this time, when $I_{1}$ and $I_{2}$ receive the request, they discard it because they have forwarded a request with the same sequence number before. On the contrary, when $I_{3}$ receives the request, it forwards it to the next hop because it never forwarded this packet previously. The increase-exclusive search policy avoids huge routing traffic and collision, caused by the flooding policy used in LAR, when the routing process failed.

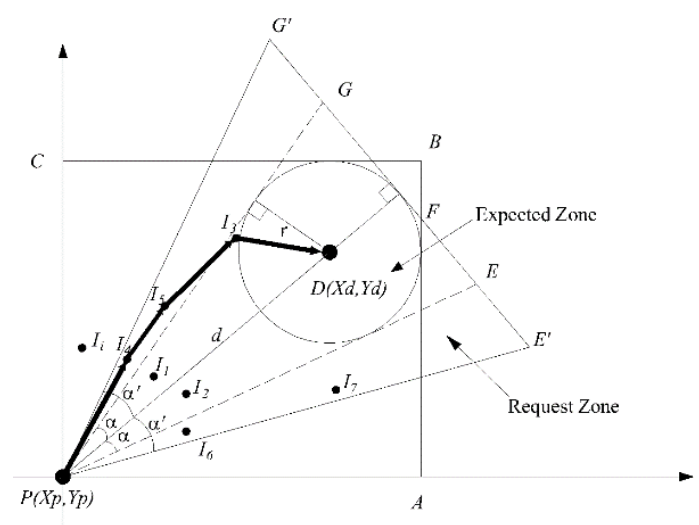

Fig.7. Resizing triangular shaped request zone

\subsection{ELLIPSE SHAPED REQUEST ZONE}

To start the route discovery we define the shape of request zone in LAR as Ellipse as shown in the following Fig.8. The distance $(x)$ between source and destination is calculated as follows: 


$$
x=\sqrt{\left(X_{d}-X_{p}\right)^{2}+\left(Y_{d}-Y_{p}\right)^{2}}
$$

Furthermore, we first define the length of major axis and minor axis of the ellipse because they determine the size of request zone [3]. The Fig.8 depicts the elliptical request zone, which is covering the expected zone as shown. Here, vertex of ellipse is chosen to be the location of source. The centre of the ellipse is $\left.\left(\left(X_{p}+X_{d}\right) / 2\right),\left(Y_{p}+Y_{d}\right) / 2\right)$, length of major axis $(l)$ is $(x+R) / 2$ and length of the minor axis $(m)$ is $r$. Here $r$ is $U \times\left(T_{1}-T_{0}\right)$.

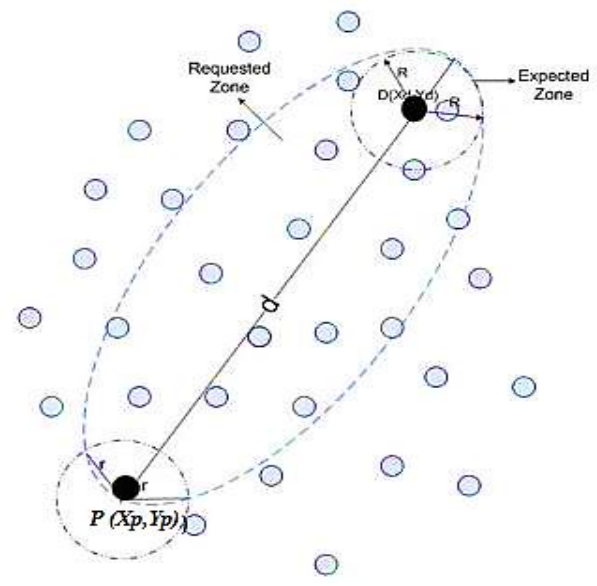

Fig.8. Ellipse shaped request zone

The request zone is calculated as the area of ellipse as follows:

$$
\text { Area }=\pi r \times \frac{x+R}{2}
$$

After defining the request zone source node sends RREQ to other nodes. Using their position information, intermediate nodes will decide about forwarding or ignoring the data received [3]. Thus by satisfying the following membership criteria a node can further propagate the packet.

$$
\frac{\left(X_{i}-X_{s}\right)^{2}}{\left(\frac{x+R}{2}\right)^{2}}+\frac{\left(Y_{i}-Y_{s}\right)^{2}}{r^{2}} \leq 1
$$

where $\left(X_{i}, Y_{i}\right)$ are the coordinates of the intermediate nodes.

Further, there is a possibility of unsuccessful route discovery in absence of path from source to destination in the defined request zone. In this scenario, the request zone should be increased to include the sufficient number of nodes to establish the path hence the protocol increases the minor axis (b) to resize the request zone. The second scenario involves collisions which lead to produce a huge routing overhead so we need to decrease the minor axis (b) of the request zone. Hence adaptive method for the adjustment of request zone where the nodes automatically adjust the parameter (minor axis) ' $b$ ' of the request zone by node densities and the required number of nodes $\left(N_{0}\right)$ inside ellipse $(E)$ are bounded by:

$$
N_{0}=\frac{\pi\left(\frac{x+R}{2}\right)}{\pi x^{2}}
$$

\subsection{DATA TRANSMISSION USING DIRECTIONAL ANTENNAS}

Radio antennas couple energy from one medium to another. An omni-directional antenna which in some cases referred as an isotropic antenna transmits or receives data equally well in all directions. On the other hand a directional antenna has certain favoured radiation and reception directions, that is, transmits/receives more energy in specific directions. Generally in MANET, motes exchange information by utilizing omnidirectional antennas [1] [4]. Therefore, data is received by all the neighbouring motes, further resulting in unintended packet receptions at the neighbouring nodes. On contrary directional antennas will radiate electromagnetic energy in specific direction and improves the coverage range. Hence less power is needed to send data using directional antenna than an omni-directional one. To reach the same receiver, a node can send the signal at a lower power focused toward the receiver instead of sending an omnidirectional signal with higher power; this reduces the energy consumption for transmission. For a given power level, a focused beam can reach greater distances than an omni-directional beam. Some receivers that are outside of omni-directional range can be reached in one hop using directional antennas. Directional antennas have a number of advantages over omni-directional antennas in ad hoc networking. By focusing energy only in the intended direction, directional antennas can increase the potential for spatial reuse and can provide longer transmission and reception ranges for the same amount of power. Increased spatial reuse and longer range translates into higher ad hoc network capacity (more simultaneous transmissions and fewer hops), and longer range also provides improved connectivity. Further, since the spatial signature of the energy is reduced to a smaller area, chances of eavesdropping are reduced, and with "smart" antennas, the steering of nulls can allow the suppression of unnecessary interference (such as jammers) at the receiver.

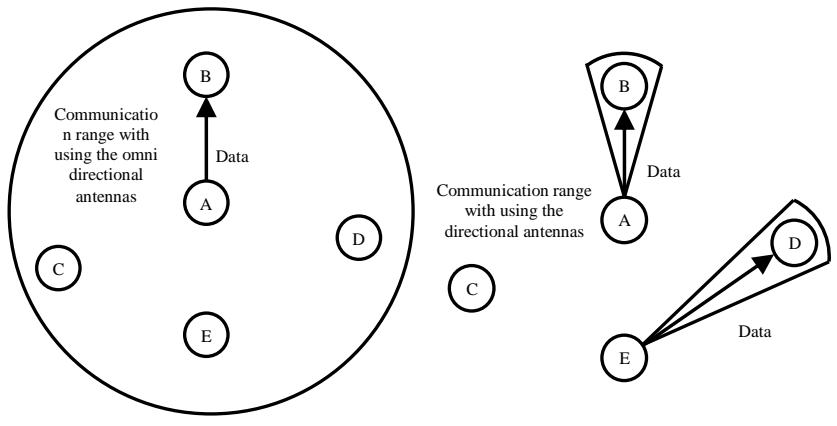

Fig.9. Comparison of omni-directional and directional antenna

\subsection{OPTIMAL ROUTE SELECTION}

Routing is a process of selecting path in a network along which the packets are sending over the network traffic. Routing protocols use routing algorithms. Job of a routing algorithm is: Given a set of routers with links connecting the routers, find a "good" path from the source to the destination. A network can be modelled by a graph by following steps:

Step 1: Routers/switches are represented by nodes.

Step 2: Physical links between routers/switches are represented by edges 
Step 3: Attached computers are ignored

Step 4: Each edge is assigned a weight representing the "cost" (speed, money, traffic) of sending a packet across that link.

The total cost of a path is the sum of the costs of the edges. The problem is to find the least-cost path. There are many routing algorithms which are used to determine the path, load, and distance over the network traffic. Routing algorithms are classified as

- Adaptive routing algorithms and

- Non-adoptive routing algorithms.

\subsubsection{Adaptive Routing Algorithm:}

It is an algorithm in which the network path can changes their routing ways in accordance to the changes taken place in the network topology and in the traffic. It is having a dynamic routing table in which it sends data over the network.

Examples: Distance vector routing algorithm, link state routing algorithm, distributed routing algorithms are comes under the category of adaptive routing algorithms.

\subsubsection{Non-Adaptive Routing Algorithm:}

These algorithms in follows a static routing table for the data to allow transmission over the network. This algorithm does not adjust with the current traffic and the network topology.

Examples: Shortest path routing, flooding algorithms are comes under the category of non-adaptive routing algorithms.

Routing algorithms that solve a routing problem based on shortest-path algorithms are Dijkstra's Algorithm, Bellman-Ford Algorithm, Floyd-Warshall Algorithm, A* Search Algorithm, Johnson's Algorithm etc.,

\subsubsection{Dijkstra's Algorithm:}

It is used to find the shortest path from one node to another node in a graph. Dijkstra's algorithm is also known as a single source shortest path algorithm. It is applied only on positive weights. The algorithm proceeds by assigning to all nodes a label which is either temporary or permanent. A temporary label represents an upper bound on the shortest distance from the home node to that node; while a permanent label is the actual shortest distance from the home node to that node. We also record information about predecessor nodes so that we may find our way along the path from the home node to the final node of the network. The paths traced out by the shortest route algorithm forms what is known as a tree structure and this is a very important concept in communications and transportation theory. Initial step: Initialise by assigning a permanent label of zero to the home node (source). All other node labels are declared to be temporary and are equal to the direct distance from the source node to that node. Select the minimum of these temporary labels and declare it permanent. In the event of a tie, choose them arbitrarily.

Step 1: Suppose that node $K$ has been assigned a permanent label most recently. Now consider the remaining nodes with temporary labels. Compare, one at a time, the temporary label of each node to the sum of the permanent label of node $K$ and the direct distance from node $K$ to the node under consideration. Assign the minimum of these distances as the new temporary label for that node. (If the old temporary label is still minimal, then it will remain unchanged during this step.)

Step 2: Select the minimum of all of the temporary labels and declare it permanent. In the case of ties, select just one of them and declare it permanent. If this happens to the destination node, then terminate. Otherwise go to step 1.

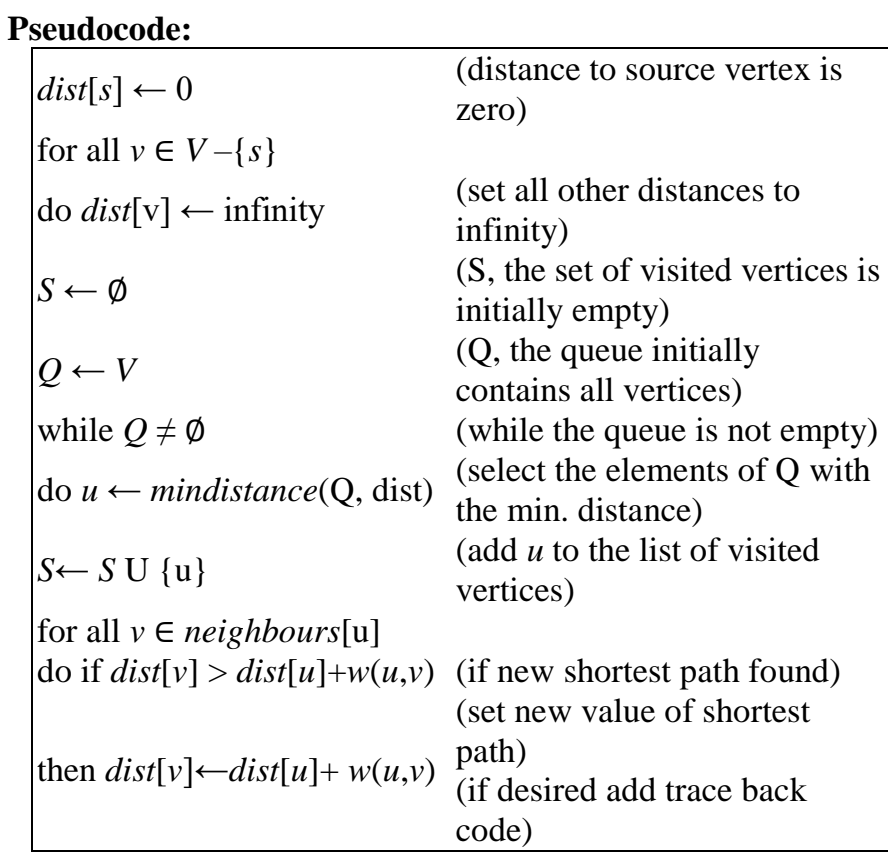

Fig.10. Pseudo code of Dijkstra's algorithm

\section{PERFORMANCE EVALUATION}

\subsection{SIMULATION MODEL}

A Multi-hop Ad hoc network with length of the 2D area over which network is deployed with length $500 \mathrm{~m}$ and width as $500 \mathrm{~m}$ is formed. It is assumed that source node $\mathrm{P}$ knows about the position of destination node $Q$ at time $T_{0}$ and at time $T_{1}$ expected zone is formed as a part of location aided routing. Also speed of all the nodes in the network is kept constant throughout the simulation. The Table. 1 provides the values of various network parameters used in our simulation.

Table.1. Simulation Parameters

\begin{tabular}{|c|c|}
\hline Parameter & Value \\
\hline Network Dimension & $500 \times 500 \mathrm{~m}$ \\
\hline Number of nodes & 70 \\
\hline Transmission range of nodes & $100 \mathrm{~m}$ \\
\hline Speed of nodes & $50 \mathrm{~m} / \mathrm{s}$ \\
\hline Elapsed time $\left(T_{1}-T_{0}\right)$ & $10 \mathrm{~s}$ \\
\hline Angle of radiation of directional antenna & 60 degree \\
\hline
\end{tabular}




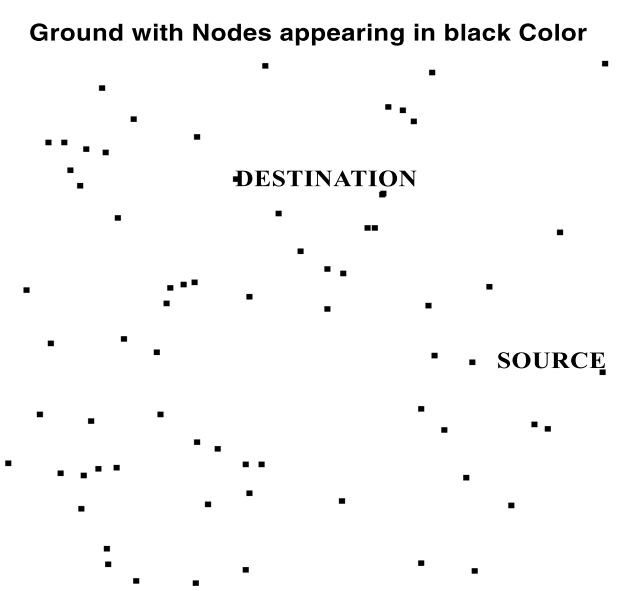

Fig.11. Network topology

\subsection{PERFORMANCE METRICS}

Evaluation of performance of various shapes of request zones and their resizing is done. Also comparison of omni-directional vs directional antennas is done based on the following metric:

\subsubsection{Routing Overhead:}

It is defined as the total number of packets propagated between all the nodes in the network during the simulation.

\section{SIMULATION RESULTS}

In this simulation, Matlab 12a software is used to evaluate the performances of various shapes of request zones. Network consisting of 70 nodes, placed randomly in a region of $500 \times 500 \mathrm{~m}$ two dimensional space is formed. Transmission range of nodes is considered as $100 \mathrm{~m}$. The Fig.12 shows the network flooding RREQ packets as a part of route discovery, without using LAR protocol and Fig. 13 with LAR using different shapes of request zones namely rectangular, teardrop, triangular, elliptical. Here RREQ packets are flooded within the request zone. One can observe the amount of routing overhead occurring in these five different scenarios. Dashed line indicates the rectangular shape and dotted lines depicts the connection between nodes which are involving in data transmission.

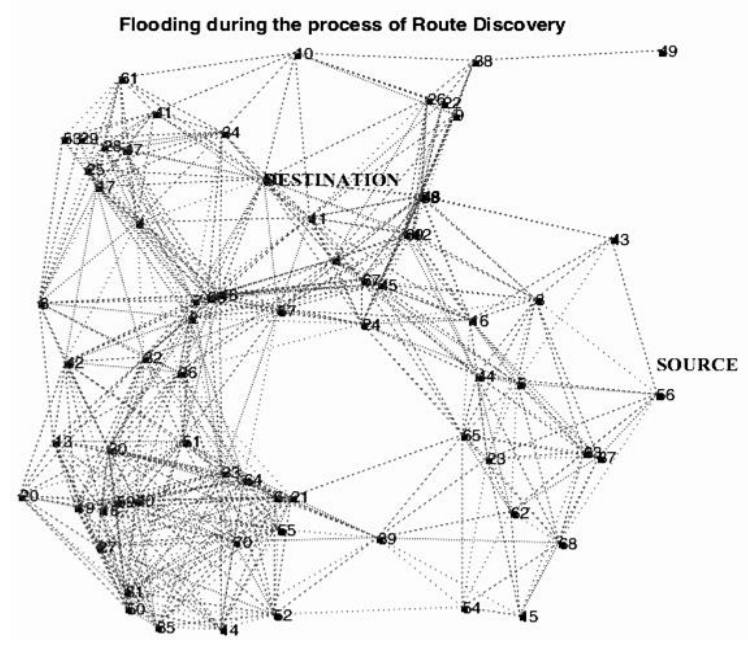

Fig.12. Flooding RREQ without using LAR protocol

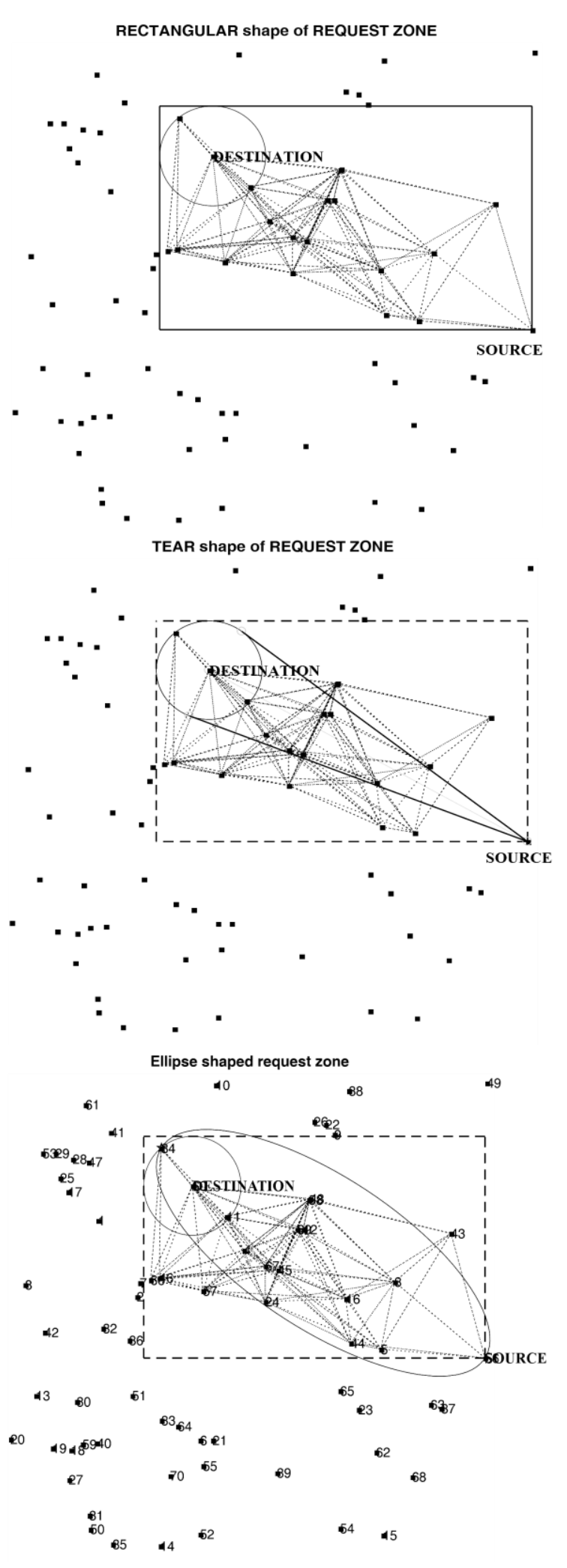




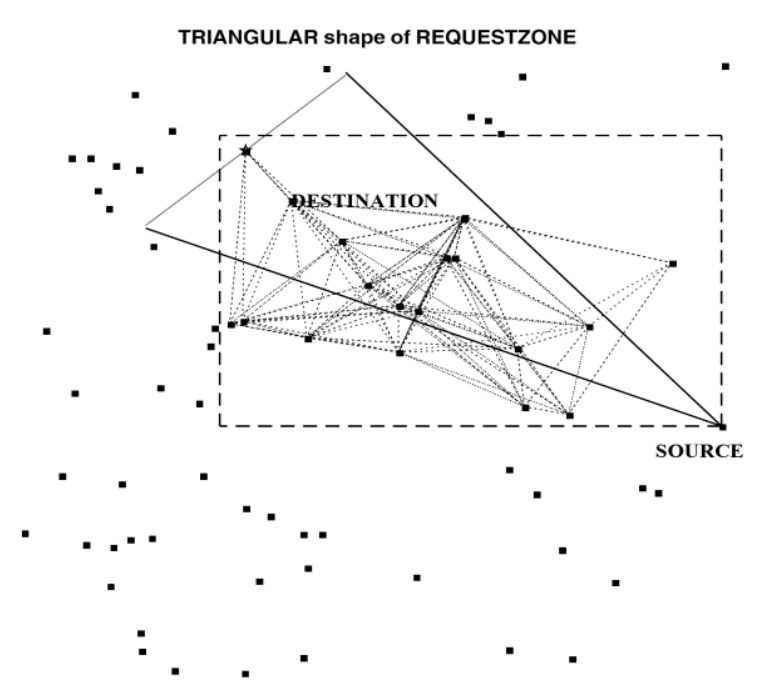

Fig.13. Flooding of RREQ packets using LAR within Rectangular, Teardrop shaped, Elliptical, Triangular request zone

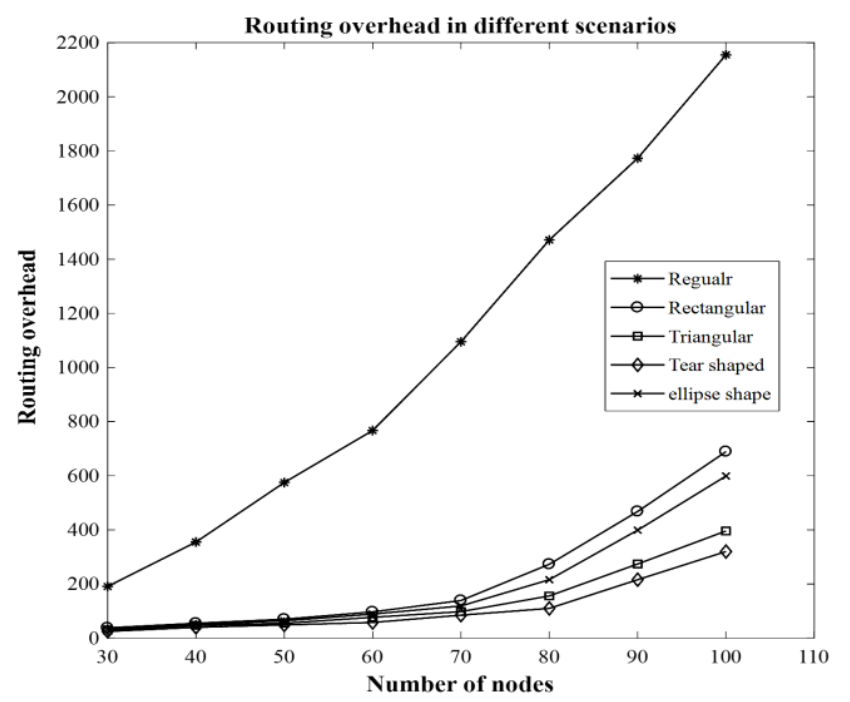

Fig.14. Comparison of routing overhead without LAR and LAR employing different shapes of request zone

Comparison between the route discovery flooding in NonLAR protocols and LAR protocol with Rectangular, Teardrop shaped, Elliptical, Triangular request zones in terms of routing overhead is presented in Fig.14. Initially when the node density is low, all shapes of request zones offer same amount of routing overhead. However network using routing protocol other than LAR i.e., regular scheme experiences more control overhead compared to LAR schemes. As density of nodes increases, it is evident that teardrop shaped request zone offers least amount of control overhead among all other shapes of request zones. Also one can observe that, performance of ellipse shaped request zone is close to rectangular request zone but offers relatively less overhead.

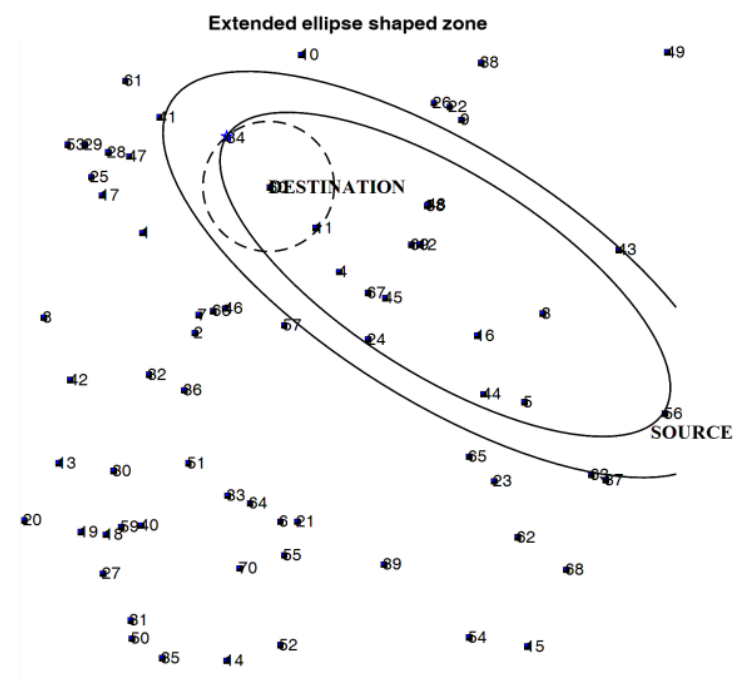

Fig.15. Resizing the elliptical shaped request zone

The Fig.15 shows how the ellipse shaped request zone has adjusted its size if route discovery fails within its earlier range.

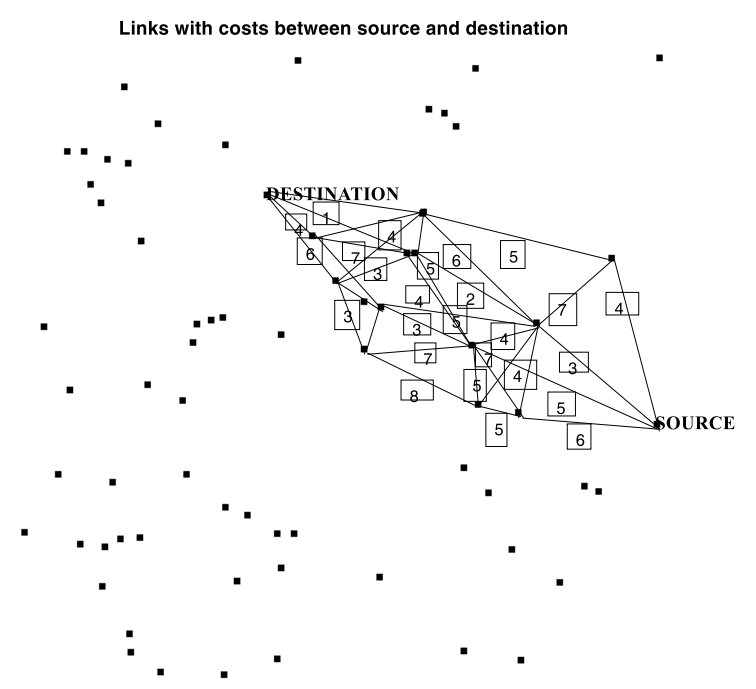

Fig.16. Network with load on links

The Fig.16 contains the network with link costs. Route which results in lowest path cost is selected using Dijkstra's algorithm. Here, after simulation the path selected is of 3 hops containing nodes numbered. 


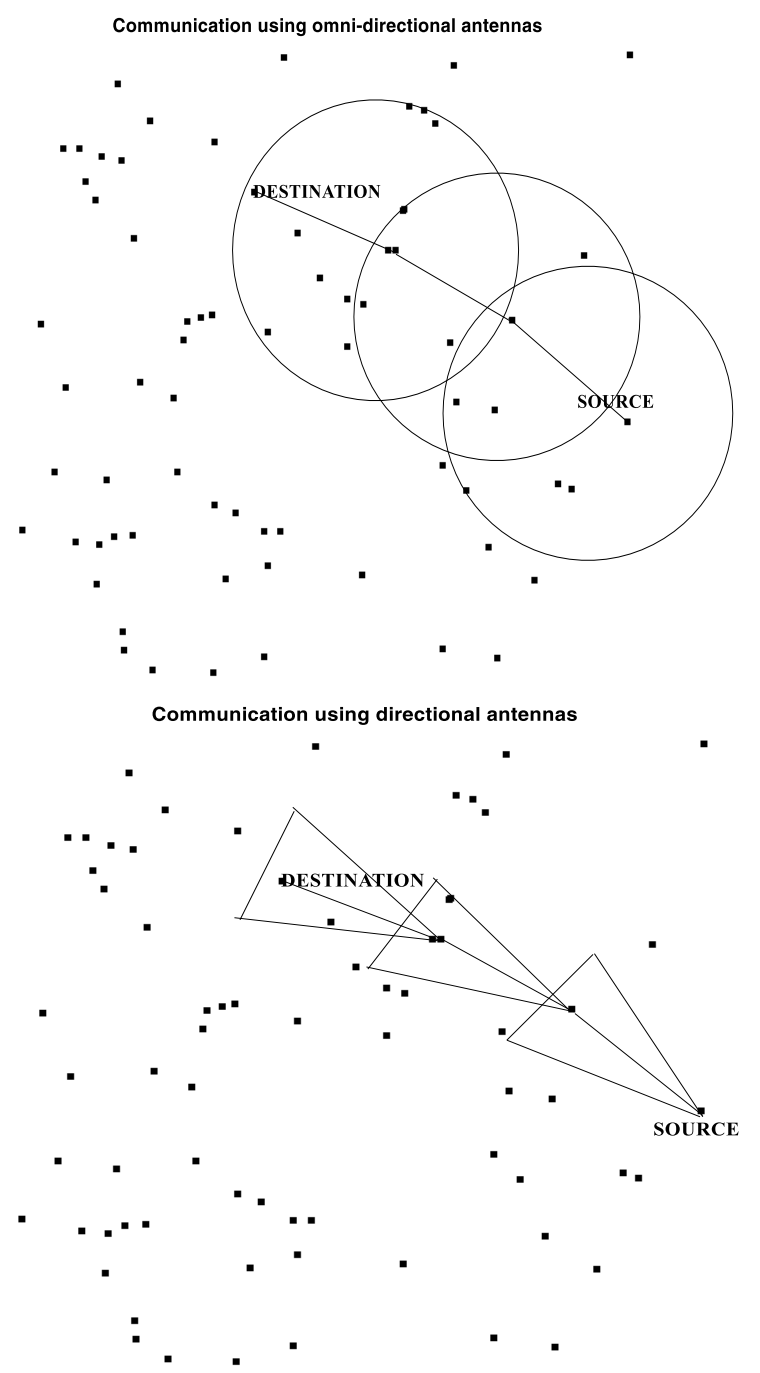

Fig.17. Network scenario using omni-directional, Directional antenna for data transmission respectively

The Fig.17 presents us the network scenario when omnidirectional, Directional antennas are used for communicating data. Here, one can observe that the number of neighboring nodes involved in data transmission in omni-directional case is more than in directional case. Now performance of directional antennas in comparison with omni-directional antennas is studied. The Fig.18 shows that numbers of nodes receiving unintended data packets are increasing significantly when omni-directional antennas are used in the network. Henceforth, packet collisions, amount of interference, latency increases. However, directional antennas are reducing redundant packet transmissions and thus provide better results outshining the performance of omnidirectional antennas.

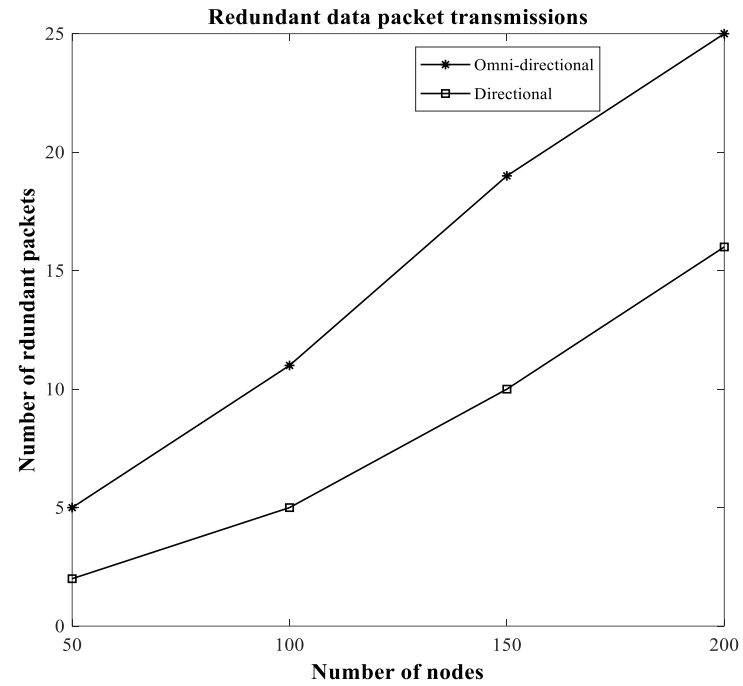

Fig.18. Comparison of omni-directional and directional antennas

\section{CONCLUSIONS AND FUTURE WORK}

From the obtained simulation results it can be concluded as, if route discovery fails by first attempt then optimal shaped request zone is resized in multi-step manner instead of extending its size to entire network. Using Dijkstra's algorithm optimal route is selected considering current status of the network with link costs indicating offered load through that link. Directional antenna outperforms omni-directional antennas by increasing transmission range of individual nodes and reduces the number of unintended packet transmissions. In the future, employing the network with varying speeds of the nodes can be included in the future work. We can extend this work to the network with bidirectional antennas in accordance with specific application. Further, energy efficient algorithm can be incorporated to enhance the protocol's performance.

\section{REFERENCES}

[1] Taku Noguchi and Takahiko Kobayashi, "Adaptive Location-Aware Routing with Directional Antennas in Mobile Adhoc Networks", Proceedings of International Conference on Computing, Networking and Communications, pp. 23-29, 2017.

[2] T.F Shih and H.C. Yen, "Location-Aware Routing Protocol with Dynamic Adaptation of Request Zone for Mobile Ad Hoc Networks", Wireless Networks, Vol. 14, No. 3, pp. 321333, 2008 .

[3] P. Mishra, S.K. Raina and B.Singh, "SDLAR: Self Adaptive Density-Based Location-Aware Routing in Mobile Adhoc Network", Proceedings of International Conference on Advanced Computing and Communication Technologies, pp. 401-419, 2016

[4] R. Ramanathan, J. Redi, C. Sant Ivanez and D. Wiggins, “Ad Hoc Networking with Directional Antennas: A Complete System Solution", Journal on Selected Areas Communications, Vol. 23, No. 3, pp. 496-506, 2005.

[5] F. De Rango, A. Lera and A. Molinaro, "Multi-Step Increase of the Forwarding Zone for LAR Protocol in Ad Hoc Networks", Proceedings of IEEE International Conference 
on Semi-Annual Vehicular Technology Conference, pp. 171176,2003

[6] Sonam Jain and Sandeep Sahu, "Topology vs Position based Routing Protocols in Mobile Ad Hoc Networks: A Survey", International Journal of Engineering Research and Technology, Vol. 1, No. 3, pp. 1-11, 2012

[7] A. Boukerche, B. Turgut, N. Aydin, M.Z. Ahmad, L. Boloni and D. Turgut "Routing Protocols in Ad Hoc Networks: A Survey", Computer Networks, Vol. 55, No. 1, pp. 30323080, 2011

[8] W.H. Liao, J.P. Sheu and Y.C. Tseng, "GRID: A Fully Location Aware Routing Protocol for Mobile Ad Hoc Networks", Telecommunication Systems, Vol. 18, No. 1-3, pp. 37-60, 2001.

[9] Xin Zhang, Xiaohong Li, Zhiyong Feng and Guangquan Xu. "Universal Analysis and Detection Framework for Location Aided Routing", Proceedings of $21^{\text {st }}$ International Conference on Engineering of Complex Computer Systems, pp. 1-7, 2016.

[10] Kamlesh Rana, Sachin Tripathi and Ram Shringar Raw, "VANET: Expected Delay Analysis for Location Aided Routing (LAR) Protocol", Proceedings of $3^{\text {rd }}$ International Conference on Computing for Sustainable Global Development, pp. 231-237, 2016

[11] Yuntao Zhua, Junshan Zhang and Kautilya Partel, "Location Aided Routing with Uncertainty in Mobile Ad Hoc Networks: A Stochastic Semi Definite Programming Approach", Mathematical and Computer Modeling, Vol. 53, No. 11-12, pp. 2192-2203, 2011.

[12] L.A Latiff, A.A Ahmed, N. Fisal and S.A. Arifin, "Directional Routing Protocol in Wireless Mobile Ad Hoc Network", Trends in Telecommunications Technologies, pp. 235-256, 2010

[13] P. Manickam, T. Guru Baskar, M. Girija and D. Manimegalai, "Performance Comparisons of Routing Protocols in Mobile Ad Hoc Networks", International Journal of Wireless and Mobile Networks, Vol. 3, No. 1, pp. 98-106, 2011.

[14] Fraser Cadger, Kevin Curran, Jose Santos and Sandra Moffett, "A Survey of Geographical Routing in Wireless Ad-Hoc Networks", IEEE Communications Surveys and Tutorials, Vol. 15, No. 2, pp. 621-653, 2012.

[15] Tomas Cakan and Vladimir Wieser, "QoS Parameters Enhancement by using Directional Antennas in MANET", Electro, pp. 26-29, 2012.

[16] N.C. Wang and S.M. Wang, "An Efficient Location Aided Routing Protocol for Mobile Ad Hoc Networks", Proceedings of $11^{\text {th }}$ International Conference on Parallel and Distributed Systems, pp. 33-38, 2005.

[17] R. Arora and N.F. Rizvi, "A Behavioural Comparison of LAR with AODV and DSR Routing Protocols", International Journal of Innovative Research in Computer and Communications Engineering, Vol. 2, No. 1, pp. 451458, 2014.

[18] Shweta R. Malwe and G.P. Biswas, "Location Aware Sector-based Routing in Wireless Ad hoc Networks", Proceedings of International Conference on Green Computing and Internet of Things, pp. 26-31, 2015.

[19] Minu A. Pillai, "Implementation of Sensor Network for Indoor Air Quality Monitoring Using CAN Interface”,
Proceedings of International Conference on Advances in Computer Engineering, pp. 72-78, 2010.

[20] Shweta R. Malwe, Soniya Rohilla and G. P. Biswas, "Location and Selective-Bordercast based Enhancement of Zone Routing Protocol", Proceedings of $3^{\text {rd }}$ International Conference on Recent Advances in Information Technology, pp. 112-118, 2016.

[21] M. Parameswaran and C. Hota, "Sector based Multicast Routing Algorithm for Mobile Ad-Hoc Networks", International Journal of Wireless and Mobile Networks, Vol. 5, No. 5, pp. 49-63, 2013.

[22] K. Wang, M. Wu, L. Weifeng, X Pengrui and S Shen, "A Novel Location Aided Routing Algorithm for MANETs", Proceedings of $5^{\text {th }}$ International Conference on Information Technology: New Generations, pp. 1162-1163, 2008.

[23] Etienne Sammut and Carl James Debono, "A Locationbased Routing Algorithm for Wireless Sensor Networks", Proceedings of IEEE International Conference on Computer as a Tool, pp. 782-786, 2015.

[24] Majid Naderi, “A New Position-Based Routing Algorithm for the Reduction of Overhead in Adhoc Networks", Proceedings of $2^{\text {nd }}$ IEEE International Conference on Systems and Networks Communications, pp. 112-119, 2007.

[25] Ruchi Aggarwal and Amanpreet Kaur, "Performance Analysis of AODV, DSDV, DSR and LAR Routing Protocols in MANET- A Review", IOSR Journal of Computer Engineering, Vol. 16, No. 3, pp. 36-42, 2014.

[26] F. De Rango, A. Molinaro, A. lera and S. Marano. "MultiStep Resizing of the Request Zone in Ad Hoc Networks", Proceedings of $14^{\text {th }}$ IEEE International Conference on Personal, Indoor and Mobile Radio Communications, pp. 62-68, 2003.

[27] Jian Shen, Wenying Zheng, Jin Wang, Zhihua Xia and Zhangjie Fu, "Routing Protocols using Directional Antennas in Ad Hoc Networks: A Comparative Review", International Journal of Grid and Distributed Computing, Vol. 6, No. 5, pp. 39-50, 2013.

[28] Hong-Ning Dai, Kam-Wing Ng, Minglu Li and Min-You $\mathrm{Wu}$, "An Overview of using Directional Antennas in Wireless Networks", International Journal of Communication Systems, Vol. 26, No. 4, pp. 413-448, 2011

[29] Peppino Fazio, Floriano De Rango and Cesare Sottile, "An On Demand Interference Aware Routing Protocol for VANETS", Journal of Networks, Vol. 2, No. 1, pp. 1-7, 2012.

[30] Takashi Watanabe, “A Receiver-Initiated Directional MAC Protocol”, Proceedings of International Conference on Communications, pp. 231-235, 2006.

[31] Hanan Saleet, Rami Langar, Otman Basir and Raouf Boutaba. "Proposal and Analysis of Region-Based Location Service Management Protocol for VANETs", Proceedings of International Conference on Global Telecommunications, pp. 12-17, 2008

[32] Sandeep Gupta and Abhishek Mathur, "Enhanced Flooding Scheme for AODV Routing Protocol in Mobile Ad Hoc Networks", Proceedings of International Conference on Electronic Systems Signal Processing and Computing Technologies, pp. 321-327, 2014.

[33] L.A. Latiff, N. Fisal, S.A. Arifin and A. Ali Ahmed, "Directional Routing Protocol in Wireless Mobile Ad Hoc 
Network", Trends in Telecommunications Technologies, 2010.

[34] A. Boukerche et al., "Routing Protocols in Ad Hoc Networks: A Survey", Computer Networks, Vol. 55, No. 13, pp. 3032-3080, 2011.

[35] N. Fisal. "Power Reduction Quadrant-based Directional Routing Protocol (Q-DIR) in Mobile Ad Hoc Network",
Proceedings of International Conference on Telecommunications, pp. 223-227, 2007.

[36] Christian Hartmann, "Hop Distances and Flooding in Wireless Multi Hop Networks with Randomized Beam Forming", Proceedings of $8^{\text {th }}$ ACM International Symposium on Modeling Analysis and Simulation of Wireless and Mobile Systems, pp. 60-72, 2005. 